\title{
In-fiber Mach-Zehnder Interferometer inscribed with femtosecond laser for high temperature sensing
}

\author{
*D. Pallarés-Aldeiturriaga ${ }^{1}$, L. Rodriguez-Cobo ${ }^{1,2}$, A. Quintela ${ }^{1,2}$, and J.M. Lopez-Higuera ${ }^{1,2}$ \\ ${ }^{1}$ Photonics Engineering Group, University of Cantabria, 39005, Santander (Spain) \\ 22CIBER-bbn, Instituto de Salud Carlos III, 28029, Madrid (Spain)
}

\begin{abstract}
In this work, an In-fiber Mach-Zehnder Interferometer has been manufactured within a standard optical fiber using direct inscription with a femtosecond laser. Different geometries have been tested in order to enhance the sensitivity of manufactured devices to high temperature sensing, reducing the total device up to $300 \mu m$. The achieved results exhibit a remarkably good response for temperature sensing up to $650^{\circ} \mathrm{C}$.
\end{abstract}

Keywords: Optical fiber sensor, high temperature, interferometer, femtosecond laser.

\section{INTRODUCTION}

Optical Fiber Sensors (OFSs) have been widely studied due to their potential application to many different scenarios. Among the different technologies in which OFSs can be based, interference between two beams highlights due to its reliability and simplicity for optical sensing. This type of OFS, known as Interferometric Fiber Optic Sensors, can be categorized in Fabry-Perot, Sagnac, Michelson and Mach-Zender Interferometers. ${ }^{1}$ Mach-Zehnder Interferometers (MZI) are of particular interest due to their high sensitivity and their final performance can be configured during the manufacturing process because their properties depend on the chosen configuration and manufacturing technique. There is a wide range of successful devices based on: Fiber tapers, collapsed/spliced PCF , open/inner air cavities, ${ }^{2,3}$ LPGs.

Another technology that can be employed to build optical sensors are femtosecond lasers that exhibit outstanding properties for optical fiber postprocessing due to low heat effects and the possibility to modify refractive index material or produce ablation just by switching pulse energy. ${ }^{4}$ Although their application on building In-fiber Mach-Zehnder Interferometers has been widely extended already, they have been limited to open/inner air cavities $^{2,3}$ having its potential to create optical waveguides unused to the best of autor knowledge.

In this work, a novel In-fiber Mach-Zehnder Interferometer based on inscribing a secondary waveguide within an optical fiber is presented and experimentally characterized for temperature sensing. Due to optical path difference, temperature dependent phase change is produced, being able to build a sensing device with a total length of $300 \mu \mathrm{m}$. The achieved results suggests a remarkably good sensor stability up to $650 \mathrm{C}$.

\section{SENSOR DESIGN}

Mach-Zender interferometers introduced in the previous section decouple a portion of light travelling into the core to the cladding where propagates after being coupled back to the core. As core and cladding modes present different effective index, optical path difference (OPD) is achieved. For the present case, light is ejected to the cladding through an inscribed waveguide near the core and couple back with the same method. This implies that several cladding modes are replaced with the induced waveguide mode, achieving different propagation lenghts for core and inscribed waveguide. Such change slightly modifies the OPD of the well known irradiance equation

*D. Aldeiturriaga: E-mail: pallaresd@unican.es, Telephone: 942200877+12 


$$
\begin{gathered}
I=I_{c o}+I_{w g}+2 \sqrt{I_{c o} I_{w g}} \cos (\phi) \\
\phi=\frac{2 \pi\left(n_{c o} L-n_{w g} L_{w g}\right)}{\lambda}+\phi_{0}=\frac{2 \pi\left(\Delta n L-n_{w g} \Delta L\right)}{\lambda}+\phi_{0} \\
F S R \approx \frac{\lambda^{2}}{\Delta n L-n_{w g} \Delta L}
\end{gathered}
$$

Where $I_{c o}$ and $I_{w g}$ represents the irradiances of the core and the waveguide respectively, $\Delta n=n_{c o}-n_{w g}$ is the difference of effective index of core and waveguide mode respectively, $\mathrm{L}$ is the sensor and $L_{w g}$ the waveguide length. FSR is the Free Spectral Range.

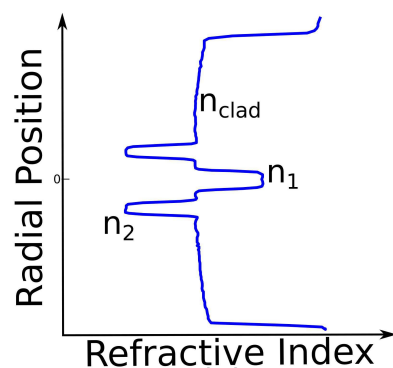

(a)

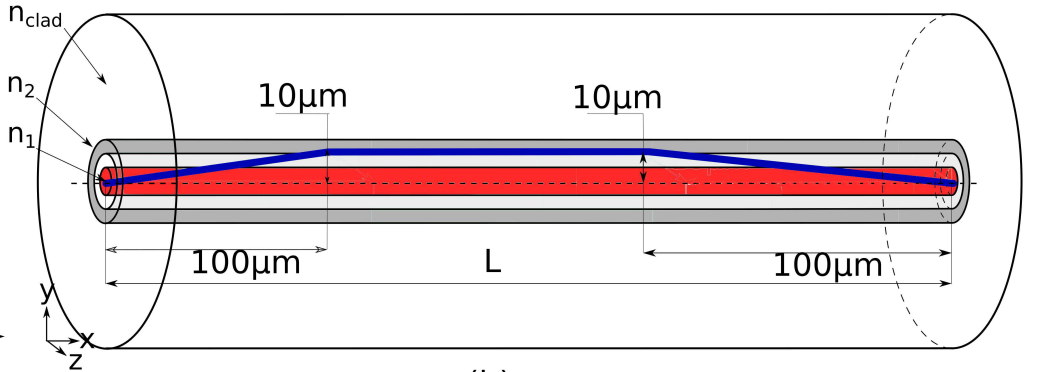

(b)

Figure 1. Waveguide inscription. Figure (a) shows the Refraction Index (RI) profile of SMF used for the sensor, (b) shows a scheme of the inscription path of the waveguide (in dark blue) in the optical fiber.

Inscription is performed in a SMF which exhibits low losses provoked by curvature thanks to its depressed cladding ring structure as shown in Fig. 1.a. Induced index change follows the path shown in the scheme of Fig. 1.b. Inscription begins at core $\left(n_{1}\right)$ and displaces $10 \mu m$ in $y$ direction (to the $n_{2}$ boundary) over $100 \mu m$ in $x$ direction in order to allow light coupling. Waveguide is partially placed inside $n_{2}$ ring until the end of inscription, where an analogue path is followed to couple back the light to the core. Sensor length $(\mathrm{L})$ is considered from the inscription start to its end, what makes path length difference $\Delta L \sim 1 \mu m$ and independent of the total device length (L).

\section{MANUFACTURING}

Aforementioned secondary waveguide introduced in previous section is performed by refractive index change, that is induced by filament glass melting due to heat accumulation effect. In order to achieve this, low writing speed and repetition rates above $50 \mathrm{kHz}$ are required. ${ }^{4}$ In this case $120 \mathrm{kHz}$ and $10 \mu \mathrm{m} / \mathrm{s}$ have been selected. Filamentation is produced below focal volume inducing positive index change while focal volume produces a negative index change for this configuration. As the focal volume does not guide light by itself, it must be placed above depressed cladding ring, this has the advantage of not requiring high precision in $z$ axis inscription compared to $x$ and $y$ axis. A capture of the inscribed waveguide by transmission microscope is shown in Fig. 2.c.

The inscription was performed by a commercial fiber laser chirp pulse amplifier (FLCPA) from CALMAR lasers operating at $1030 \mathrm{~nm}, 1.09 \mu \mathrm{J}$ pulse energy, 370fs pulse duration, focused through a $\mathrm{NA}=0.5$ objective lens from Mitutoyo to a commercial stripped SMF (BendBright XS from Draka Comteq). The sample was hold over a nano resolution X-Y-Z linear motor stage from Aerotech. During inscription, the sample was illuminated by a HP Broadband light Source 83437A and its spectra gathered by a HP Optical Spectrum Analyzer 86142A.

Fig 2.a shows the crossection of the optical fiber at the middle of the structure illuminated by a white light source. The focal region at $38 \mu \mathrm{m}$ below surface can be noticed (Fig. 2.a) and also a small filament below guiding light at the depressed cladding ring at $10 \mu \mathrm{m}$ from the center. Images captured by a CCD camera of both sensor start/end focused at $38 \mu \mathrm{m}$ below fiber are depicted in Fig. 2.b. 


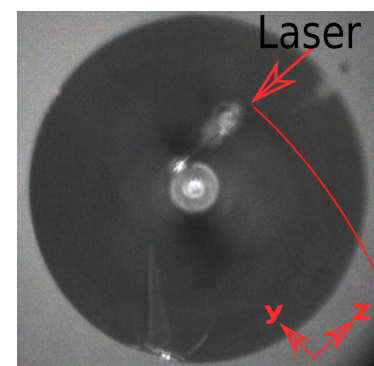

(a)
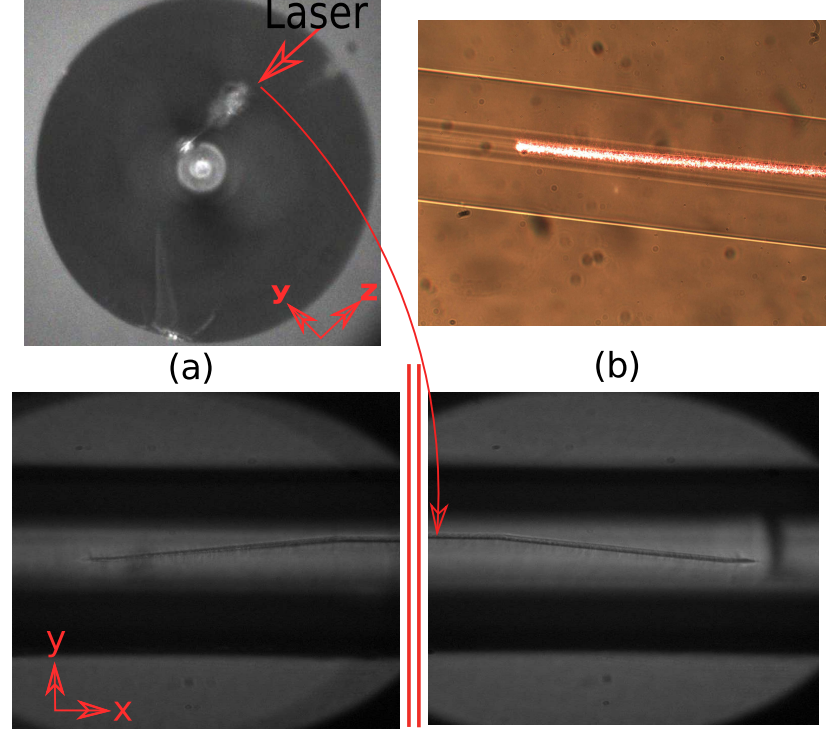

(b)

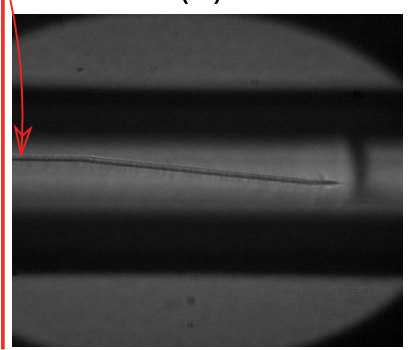

(c)

Figure 2. Manufacturing result. (a) is a Capture of the inscribed waveguide by transmission microscope, optical fiber was iluminated by a red laser source and submerged in index matching oil. A view of the fiber section at the middle of the inscription is shown in (b) while white light propagates inside . (c) shows both inscriptions arms focused at $38 \mu m$ below fiber surface. All the images have been captured by a CCD camera.

This process was repeated with lengths from $3 \mathrm{~mm}$ to $0.3 \mathrm{~mm}$, their transmission spectra are represented in Fig. 3. High lengths drops transmission and FSR due to waveguide loss and path difference increase, as shown in Eq. 3. Loss can be mainly provoked by the lack of beam correction and adaptive optics for the cylindrical geometry, ${ }^{5}$ what makes shorter devices preferred for manufacturing. At $0.3 \mathrm{~mm}$, the spectrum exhibits only one clear valley, thus, consequently further experimental tests will be performed for this geometry.

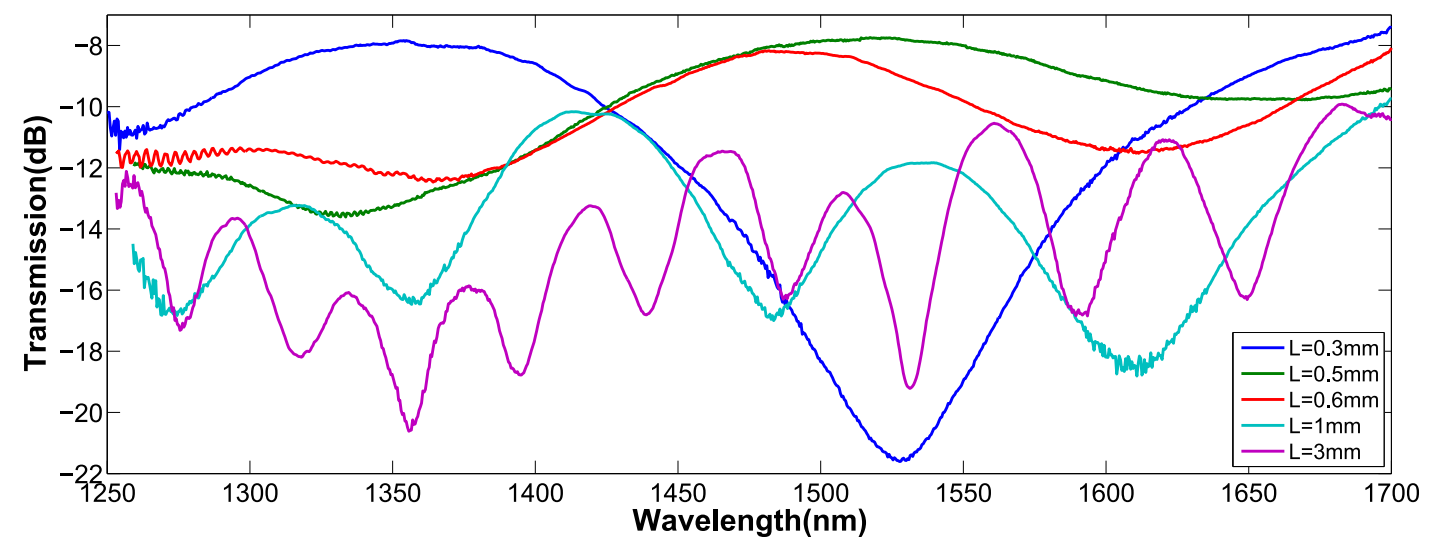

Figure 3. Transmission spectra comparison between devices of different lengths.

\section{EXPERIMENTAL CHARACTERIZATION}

Temperature characterization of $\mathrm{L}=0.3 \mathrm{~mm}$ sample was performed by heating the sample with a Carbolite tubular furnace. Starting from $50{ }^{\circ} \mathrm{C}$, temperature was increased in steps of $50{ }^{\circ} \mathrm{C}$ averaging its spectra over 10 minutes for every temperature up to $650{ }^{\circ} \mathrm{C}$. Transmission spectra for temperatures up to $650{ }^{\circ} \mathrm{C}$ are represented in Fig. 4.a. As temperature increase, the wavelength of the resonance decreases. 


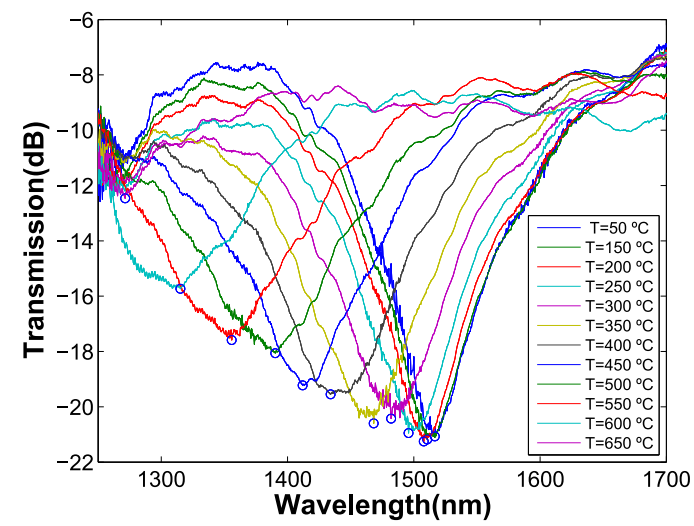

(a)

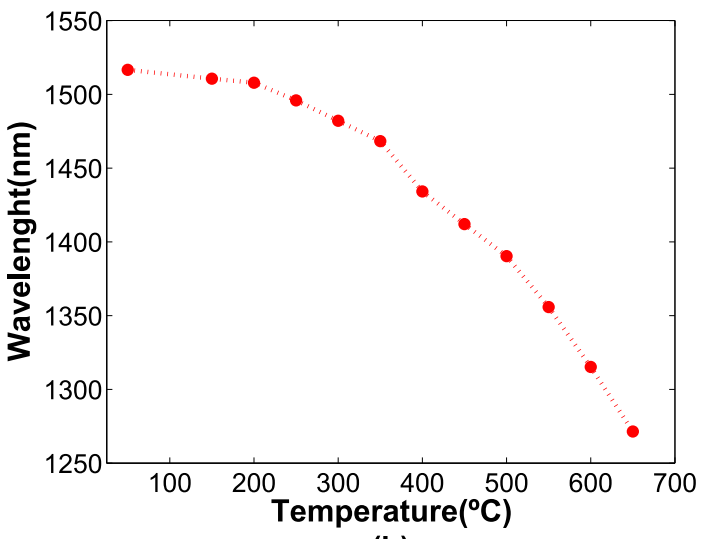

(b)

Figure 4. Selecting $\mathrm{L}=0.3 \mathrm{~mm}$, (a) plots several transmission spectra at different temperatures ranging from $50^{\circ} \mathrm{C}$ to $650^{\circ} \mathrm{C}$ while (b) plots its wavelength minima.

For higher temperatures than $650{ }^{\circ} \mathrm{C}$ transmission peak shifts below emitted spectra and loses its interferometric shape. Wavelength of the minimum in function of Temperature is depicted in Fig. 4.b. The sensitivity increases at higher temperatures that suggest changes in the index change provoked during the inscription.

\section{CONCLUSION}

An In-fiber Mach-Zehnder Interferometer based on femtosecond waveguide inscription is presented. Due to its lack of adaptive optics and beam corrections, this sensor has a remarkable easy manufacturing with a length as short as $300 \mu \mathrm{m}$ taking advantage of the filamentation process. Previous works of In-fiber Mach-Zehnder manufactured by femtosecond lasers achieve more compact structures but they require ablation or void formation that can deteriorate the mechanical response of the device. Temperature characterization shows high sensitivity between $450^{\circ} \mathrm{C}$ and $650^{\circ} \mathrm{C}$ that suggests certain optical degradation that has been verified in different devices. Further tests will be performed to achieve a better characterization of this phenomenon.

\section{ACKNOWLEDGMENTS}

This work has been supported by the projects TEC2013-47264-C2-1-R, TEC2016-76021-C2-2-R and grant BES-2014-069736 of the Spanish government and by the Parliament of Cantabria postdoc grant.

\section{REFERENCES}

[1] Lee, B. H., Kim, Y. H., Park, K. S., Eom, J. B., Kim, M. J., Rho, B. S., and Choi, H. Y., "Interferometric fiber optic sensors," Sensors 12(3), 2467-2486 (2012).

[2] Hu, T. Y., Wang, Y., Liao, C. R., and Wang, D. N., "Miniaturized fiber in-line Mach-Zehnder interferometer based on inner air cavity for high-temperature sensing.," Optics letters 37(24), 5082-5084 (2012).

[3] Liu, Y., Li, M., Sun, H., Li, Y., and Qu, S., "Ultrasensitive liquid refractometer based on a Mach-Zehnder micro-cavity in optical fibre fabricated by femtosecond laser-induced water breakdown," Journal of Modern Optics 63(21), 2285-2290 (2016).

[4] Tan, D., Sharafudeen, K. N., Yue, Y., and Qiu, J., "Femtosecond laser induced phenomena in transparent solid materials: Fundamentals and applications," Progress in Materials Science 76, 154-228 (2016).

[5] Osellame, R., Cerullo, G., and Ramponi, R., [Femtosecond Laser Micromachining Photonic and Microfluidic Devices in Transparent Materials], Springer Berlin Heidelberg, Berlin, Heidelberg (2012). 